> Un stress de faible intensité peut avoir des effets bénéfiques sur l'organisme en induisant une réaction de surcompensation après une perturbation de l'homéostasie. Certains stress modérés augmentent la longévité et améliorent le vieillissement chez la drosophile. Les études sur les rongeurs sont rares, mais il a été montré chez les humains que, par exemple, une insuffisance cardiaque permet une meilleure survie en cas d'infarctus. D'autres études, dans des situations moins dramatiques, suggèrent que le stress modéré a des effets positifs au cours du vieillissement. Le stress modéré est peut-être une piste intéressante en thérapie ou pour la prévention des effets négatifs du vieillissement, et il serait nécessaire de développer les études chez l'humain. <

Comment vivre en bonne santé le plus longtemps possible ? Il s'agit d'une question cruciale des sociétés modernes, car personne ne souhaite que l'augmentation de l'espérance de vie actuellement observée (un peu plus de deux mois chaque année en France) se traduise par une plus longue période en mauvaise santé à la fin de la vie. Heureusement, l'espérance de vie en bonne santé s'accroît, même si la France occupe un rang moyen parmi les pays européens, alors qu'elle est plutôt en tête pour l'espérance de vie à la naissance [1]. Les progrès dans le traitement des maladies cardiovasculaires ou autres, de l'hygiène de vie, du suivi des personnes âgées expliquent que vieillesse rime moins, ou de plus en plus tard, avec détresse.

Peut-on faire mieux? Autre question cruciale, et on pense par exemple au traitement de la maladie d'Alzheimer ou d'autres maladies liées à l'âge. À côté de ces pistes «traditionnelles », le propos de cet article est d'inciter médecins et chercheurs à la réflexion sur une piste thérapeutique éventuelle, peu explorée jusqu'ici : I'utilisation du stress modéré. Cet article décrit les résultats de la recherche chez la mouche du vinaigre, insecte chez lequel ont été accumulés le plus de résultats, avant de s'intéresser aux

\section{La modulation du vieillissement par le stress modéré}

\section{De la mouche aux humains?}

Éric Le Bourg

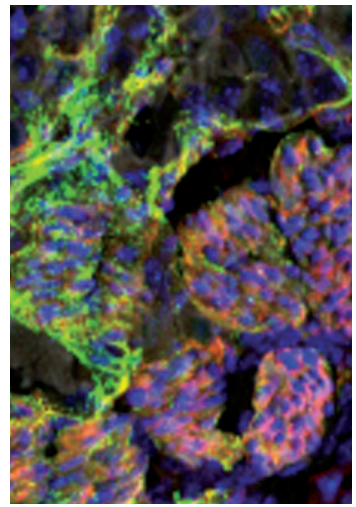

Université Paul-Sabatier, centre de recherche sur la cognition animale, UMR CNRS 5169,

118 , route de Narbonne, 31062 Toulouse Cedex 9, France. lebourg@cict.fr

rongeurs et aux humains, et de montrer l'intérêt possible du stress modéré en thérapie.

Depuis plus d'un siècle, les toxicologistes ont montré que de faibles doses de produits toxiques avaient des effets de stimulation par exemple sur la croissance des plantes ou des bactéries [2]. L’hypothèse de l'hormésis (ou hormèse) suggère que des agents stressants de faible intensité peuvent avoir des effets bénéfiques sur l'organisme en induisant une réaction de surcompensation en réponse à une perturbation de l'homéostasie provoquée par l'agent stressant. Ces effets, dits hormétiques, ne sont pas limités à la toxicologie puisque, par exemple, un choc thermique à $34^{\circ} \mathrm{C}$ peut aider des larves de mouches à résister par la suite plus longtemps à une exposition longue à $0^{\circ} \mathrm{C}$ [3]. Depuis une vingtaine d'années, l'idée qu'un stress modéré pourrait permettre d'améliorer le vieillissement a donné lieu à un certain nombre d'expériences.

\section{Le stress modéré chez la mouche}

La drosophile (Drosophila melanogaster) utilisée en génétique depuis plus d'un siècle l'est aussi en biologie du vieillissement du fait de ses avantages : durée de développement de 9 jours, longévité à $25^{\circ} \mathrm{C}$ de l'ordre de 3 mois, coûts d'élevage faibles, étude du comportement et de l'apprentissage possible, etc.). Sa faible taille (2 $\mathrm{mm}$ ) permet l'élevage d'un grand nombre d'animaux dans un volume réduit et la conduite d'expériences avec de grands effectifs, ce qui n'est généralement pas possible avec les rongeurs. Le Tableau / résume la majeure partie des études relativement complètes (effets sur la longévité, le vieillissement comportemental, la résistance aux stress forts). 
L'hypergravité, un niveau de gravité supérieur à la gravité terrestre, a été utilisée comme stress modéré après que de premières études aient révélé qu'une exposition tout au long de la vie adulte diminuait la longévité et accélérait le vieillissement comportemental [4]. Si l'hypergravité (jusqu'à 5 ou 7 fois la gravité terrestre) était un stress fort dans ces conditions, ne l'appliquer que deux semaines en début de vie pourrait s'apparenter à un stress modéré dont les effets seraient positifs. En effet, l'hypergravité appliquée en début de vie augmente la longévité des mâles d'environ $10 \%$, celle des femelles pouvant toutefois ne pas être modifiée. Les mouches des deux sexes résistent plus longtemps à une exposition létale à $37^{\circ} \mathrm{C}$ et leur vieillissement comportemental peut être retardé. Les mâles survivent aussi plus longtemps à une simulation de la canicule, c'est-à-dire à des chocs thermiques non létaux répétés qui diminuent la longévité. En revanche, l'hypergravité n'a pas d'effet sur la résistance au froid, au peroxyde d'hydrogène, à la dessiccation ou à l'infection fongique, mais elle diminue la résistance à l'inanition. Une synthèse accrue de protéines de choc thermique de $70 \mathrm{kDa}$ en cas de coup de chaleur pourrait expliquer la meilleure résistance à la chaleur, mais pas l'effet sur la longévité (ces protéines n'étant pas synthétisées à $25^{\circ} \mathrm{C}$, la température d'élevage des mouches). L'activité de deux enzymes antioxydantes (superoxyde dismutase et catalase) n'est pas modifiée par l'hypergravité, ce qui pourrait expliquer l'absence d'effet de ce stress sur la résistance au stress oxydant [4-6].

Un autre stress modéré, l'exposition à la chaleur, s'est accompagné d'effets variables selon les souches de mouches et les procédures utilisées. Les conclusions sont les suivantes : les coups de chaleur (1) augmentent légèrement la longévité, ainsi que la résistance à la chaleur létale et au froid ; (2) ont des effets variables sur le vieillissement comportemental et la résistance au stress oxydant; (3) n'ont pas d'effet sur la résistance à l'infection fongique et (4) diminuent la résistance à l'inanition (revue dans [7]).

Le froid, sous la forme d'une exposition d'une heure par jour à une température de $0{ }^{\circ} \mathrm{C}$ pendant les deux premières semaines de la vie adulte: (1) augmente la longévité des mâles (et parfois celle des femelles) et la résistance à différents stress forts à différents âges (température, froid, canicule, infection fongique chez les mâles); (2) et retarde une forme de vieillissement comportemental (Tableau I). Toutefois, le froid a des effets négatifs sur la résistance à l'inanition (au moins chez les femelles), peut diminuer fortement la longévité des femelles, et n'a pas d'effet clair sur la résistance au stress oxydant [6, 8]. Par ailleurs, exposer les mouches au froid à des âges plus avancés permet aussi d'observer des effets positifs surtout chez les mâles, mais les effets sur la longévité et la résistance à l'infection fongique (Figure 1) peuvent disparaître si cette exposition a lieu à un âge très tardif [9].

On voit que, chez la drosophile, c'est le froid qui a les effets positifs les plus prononcés, puisque la longévité peut augmenter, une forme de vieillissement comportemental être retardée, et la résistance à la chaleur, à l'infection et à d'autres stress être accrue (ces effets étant observés y compris à des âges avancés). Un autre modèle d'invertébré a été utilisé pour analyser les effets du stress modéré. II s'agit du ver Caenorhabditis elegans, chez lequel a été analysée l'exposition à la chaleur, au rayonnement gamma, ou encore à des produits toxiques. Les résultats ont montré le plus souvent une augmentation de la longévité ou de la résistance au stress (revue dans [7]).

Reste la question des mécanismes par lesquels agit un stress modéré. Jusqu'à maintenant il a été possible d'éliminer le rôle de certains acteurs biochimiques tels que les enzymes antioxydantes et de relativiser celui des protéines de choc thermique, sans pour autant pouvoir proposer un mécanisme principal (revue dans [7]). Le fait que des stress modérés variés puissent avoir des effets relativement semblables, y compris des stress inconnus dans la nature comme l'hypergravité, suggère que toute perturbation de l'homéostasie liée au stress pourrait déclencher une cascade de réponses impliquant, par exemple, des facteurs de transcription. La recherche sur les mécanismes du stress modéré sera probablement une priorité dans les années à venir.

\section{Le stress modéré chez les rongeurs}

Très peu d'études expérimentales ont été réalisées chez les rongeurs. En revanche, il est connu depuis longtemps que l'activité physique peut augmenter leur longévité. L'exercice volontaire, et donc modéré, a des effets positifs sur la longévité moyenne (+ $10 \%)$, mais pas obligatoirement l'exercice forcé qui est plus intense [10, 11]. L'exercice peut améliorer le vieillissement comportemental [12], les fonctions immunitaires [13] et diminuer les maladies rénales [14] chez les rats âgés. Comme l'exercice augmente la production des radicaux libres par l'intermédiaire d'un métabolisme plus élevé et donc d'une consommation d'oxygène plus forte [15], l'exercice peut être considéré comme un stress modéré et ses effets positifs sur le vieillissement et la longévité sont maintenant souvent réinterprétés comme étant des effets hormétiques.

Par ailleurs, plonger des rats âgés de 9 à 32 mois dans une eau à $23^{\circ} \mathrm{C}, 4 \mathrm{~h}$ par jour, 5 jours par semaine n'a pas d'effet significatif sur la longévité, diminue l'incidence des sarcomes et des carcinomes, mais augmente les pathologies cardiovasculaires [16]. D'autres études utilisant des préparations de cœur isolé seront décrites dans le paragraphe suivant.

\section{Le stress modéré chez les humains}

Il est maintenant connu que l'activité physique a des effets positifs chez les humains. Les personnes ayant une activité physique régulière vivent plus longtemps puisque, dans un groupe de près de 17000 personnes 


\begin{tabular}{|c|c|c|c|c|c|c|}
\hline & \multicolumn{2}{|c|}{ Chaleur } & \multicolumn{2}{|c|}{ Froid } & \multicolumn{2}{|c|}{ Hypergravité } \\
\hline & Mâles & Femelles & Mâles & Femelles & Mâles & Femelles \\
\hline Longévité & + & + & + & + ou 0 & + & 0 \\
\hline Activité locomotrice & + ou 0 & + ou 0 & Non étudié & Non étudié & 0 & 0 \\
\hline Activité d'ascension & 0 & 0 & + & + & + & + ou 0 \\
\hline Chaleur létale & + & + & + & + & + & + \\
\hline «Canicule» & Non étudié & Non étudié & + & + & + & 0 \\
\hline Dessiccation & Non étudié & Non étudié & Non étudié & Non étudié & 0 & 0 \\
\hline Froid & + & + & + & + & 0 & 0 \\
\hline Inanition & - & - & 0 & - & - & - \\
\hline Peroxyde d'hydrogène & 0 & 0 & 0 & 0 & 0 & 0 \\
\hline Paraquat & + & + & Non étudié & Non étudié & Non étudié & Non étudié \\
\hline Infection fongique & 0 & 0 & + & 0 & 0 & 0 \\
\hline
\end{tabular}

Tableau I. Résumé des effets de trois stress modérés sur la longévité, le vieillissement comportemental et la résistance à différentes agressions de la drosophile. Un + indique qu'un effet positif a généralement été observé (par exemple, une plus grande longévité), un - un effet négatif (par exemple, une moins bonne résistance à l'inanition), un 0 une absence d'effet. Les résultats variables d'une expérience à l'autre sont indiqués par « + ou 0 ». L'activité locomotrice est une mesure du déplacement spontané ; l'activité d'ascension est la capacité à gravir une paroi verticale; la chaleur létale est l'exposition à $37{ }^{\circ} \mathrm{C}$ jusqu'à la mort des animaux; la canicule est l'exposition répétée à $37^{\circ} \mathrm{C}$ pour une durée non létale mais qui diminue la longévité d'environ $50 \%$ par la suite ; la résistance au stress oxydant est mesurée par le temps de survie après exposition au peroxyde d'hydrogène ou au Paraquat, un herbicide. Toutes ces études sont référencées dans [7].

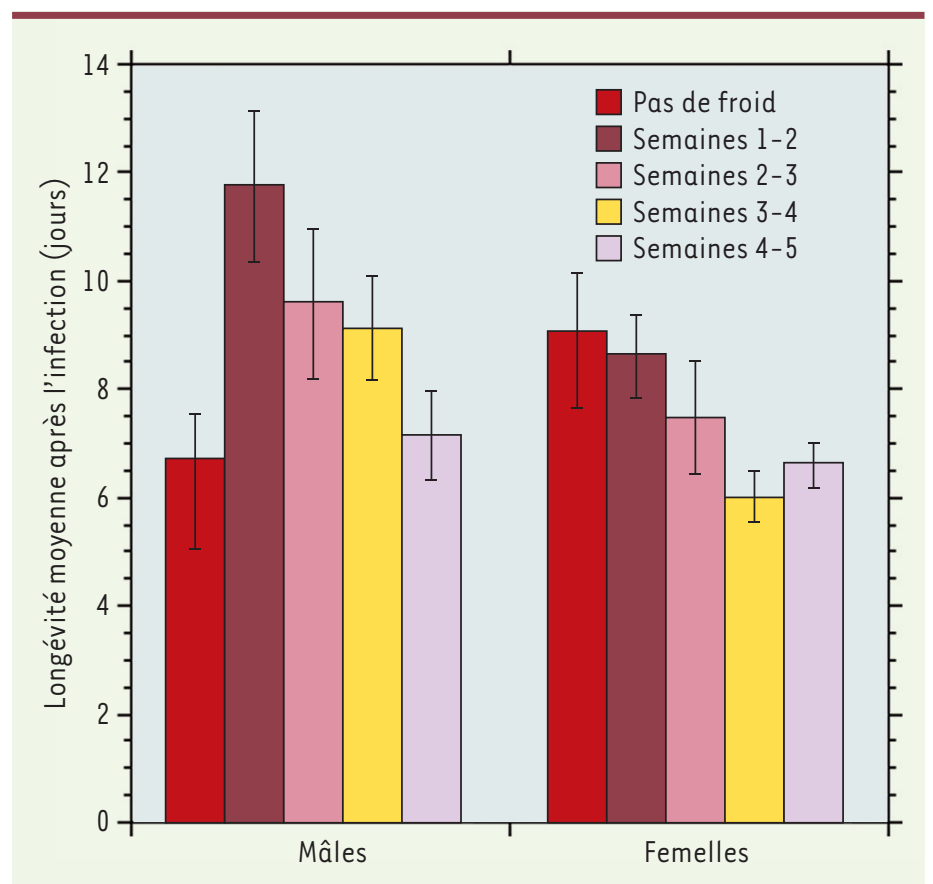

Figure 1. Effet d'un prétraitement par le froid sur la longévité après une infection. La longévité moyenne a été étudiée après une infection à l'âge de 6 semaines par le champignon Beauveria bassiana chez des mouches prétraitées à différents âges. Le prétraitement consiste en une exposition au froid $\left(0^{\circ} \mathrm{C}\right)$ d'une heure par jour, cinq jours par semaine pendant deux semaines. Les résultats représentent la longévité moyenne \pm l'erreur standard (d'après [9]). âgées de 35 à 79 ans et suivies pendant 12 à 16 ans, une telle activité permet d'accroître la vie de plus d'une année supplémentaire par rapport à un groupe inactif. Cet effet bénéfique s'explique en particulier par une plus faible mortalité due aux maladies cardiovasculaires ou respiratoires et, dans une moindre mesure, aux cancers [17]. De plus, dans une étude d'une cohorte d'hommes âgés de 45 à 84 ans en 1977, suivis pendant 11 ans ou jusqu'à l'âge de 90 ans, l'adoption d'un style de vie actif augmente la longévité d'environ 18 mois, alors que la diminution de l'activité physique la réduit de presque 3 ans [18]. Une méta-analyse de 18 études individuelles précédemment publiées rapporte que l'activité physique améliore les fonctions cognitives des personnes de 55 à 80 ans, et en particulier celle des femmes [19]. Une activité physique modérée peut donc avoir des effets positifs, en particulier sur le vieillissement, bien qu'une activité trop intense puisse s'accompagner d'effets négatifs. Comme chez les rongeurs, les effets de l'activité physique chez l'humain peuvent être interprétés comme étant des effets hormétiques. Le stress modéré a une influence positive sur les pathologies cardiaques. Ainsi, si on expose un cœur isolé de rat à un bref épisode d'ischémie suivi d'une reperfusion (préconditionnement ischémique), les dommages provoqués par une ischémie plus sévère 


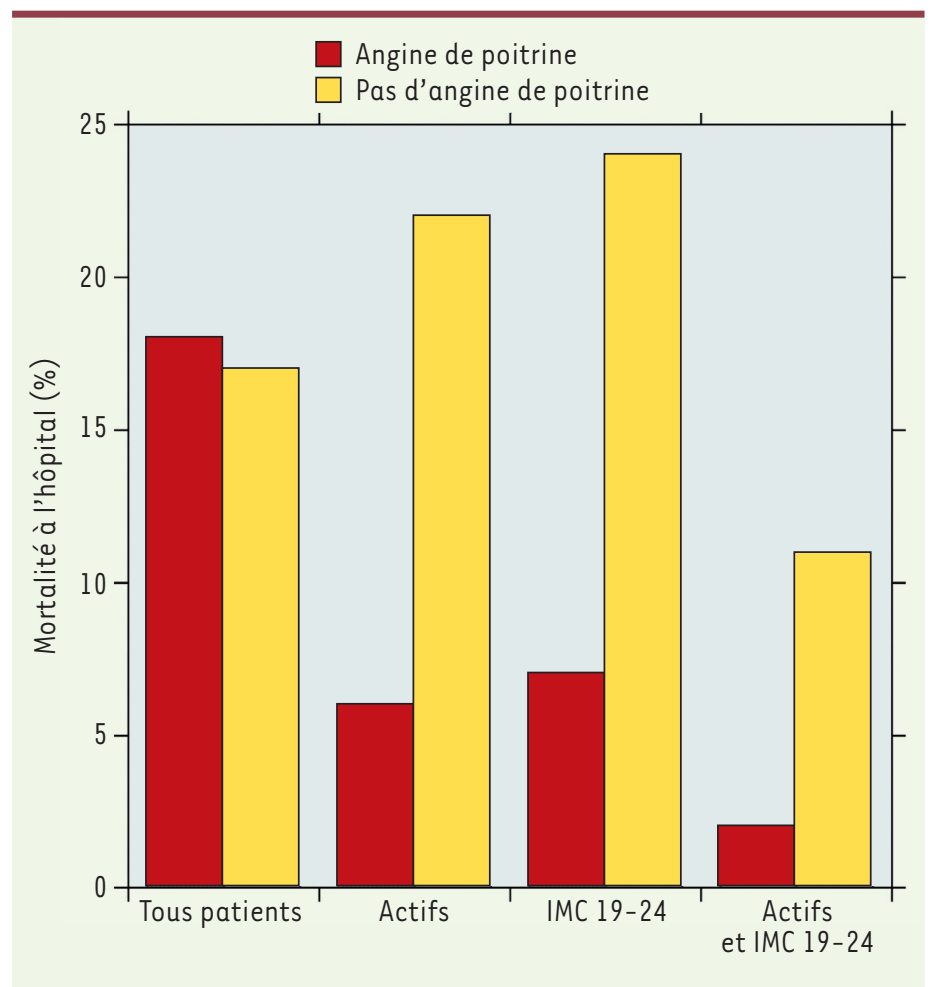

Figure 2. Effets de l'angine de poitrine préalable, de l'activité physique, et de la morphologie de patients âgés de 75 ans en moyenne sur la mortalité à l'hôpital après un infarctus du myocarde. Les patients sveltes ont un indice de masse corporelle (IMC) compris entre 19 et $24 \mathrm{~kg} / \mathrm{m}^{2}$ (d'après [21]).

seront réduits, mais cet effet disparaît chez les animaux âgés [20]. Cette dernière situation est réversible, puisque soumettre les rats âgés à une diminution de leur ration alimentaire ou à l'exercice physique restaure l'effet du préconditionnement ischémique, l'effet étant plus important si les animaux sont soumis aux deux traitements.

Chez l'homme, l'analyse clinique des patients souffrant d'angine de poitrine révèle une mortalité plus faible en cas d'infarctus [21], mais cet effet positif disparaît chez le patient âgé (Figure 2). Les patients âgés et sveltes (évitant probablement une alimentation trop riche) conservent toutefois cet effet positif de l'angine de poitrine, de même que ceux qui exercent une activité physique élevée. Enfin, combiner une forte activité physique et être svelte renforce les effets positifs d'une angine de poitrine préalable. Ces résultats sont en accord avec ceux qui sont observés dans les préparations de cœur isolé de rat. Pour survivre à un infarctus en l'absence d'angine de poitrine, il est donc préférable pour une personne âgée d'être svelte et active, confirmant que le poids excessif et la vie sédentaire sont des facteurs aggravant les maladies cardiovasculaires $[17,22]$.

Les effets positifs du stress modéré ont été observés dans d'autres situations dramatiques. Ainsi, le fait d'avoir eu dans un passé récent un accident ischémique cérébral de nature transitoire, c'est-à-dire la perte brutale d'une fonction cérébrale ou oculaire pendant une durée brève (moins d'une heure), diminue la gravité d'un infarctus cérébral, tant sur le plan de la récupération fonctionnelle que du risque de décès [23].

Les effets positifs du stress modéré ont été également rapportés chez des patients où le pronostic vital n'était pas engagé. Ainsi, des séances de sauna - 15 min à $60^{\circ} \mathrm{C}$ - ont fait diminuer en deux semaines la tension artérielle et la glycémie de personnes atteintes de problèmes cardiaques, et le poids de personnes obèses [24].

\section{Perspectives thérapeutiques du stress modéré chez l'homme}

Tous ces résultats montrent que le stress modéré a des effets positifs chez l'humain, y compris dans des situations critiques comme la survenue d'un infarctus. Cependant, chez le sujet âgé, ces effets ne sont observés que dans certaines conditions. Les études chez la mouche montrent que les effets du stress modéré peuvent être durables, puisqu'ils augmentent la longévité et la résistance à différents stress à des âges avancés, longtemps après la fin d'un prétraitement (par le froid par exemple, voir Figure 1). Des stress modérés chez l'humain pourraient-ils s'accompagner d'effets à long terme (pendant des semaines, des mois ou des années) dans la vie quotidienne et ne pas se restreindre aux seuls effets transitoires dans des situations critiques comme l'ischémie cérébrale $[25,26]$ ?

Par exemple, il a été observé qu'un traitement par la chaleur de synoviocytes (cellules qui produisent le liquide synovial) provenant de patients souffrant d'arthrite rhumatoïde diminuait l'activation de gènes codant pour des protéines pro-inflammatoires [27]. Pourrait-on imaginer de soumettre les articulations de patients souffrant d'arthrite à une hyperthermie ou même, en prévention, pratiquer de telles hyperthermies chez le sujet sain? Le débat sur l'efficacité des cures thermales est presque aussi vieux que leur existence, mais une étude récente semble indiquer l'efficacité d'une cure thermale dans le traitement de l'arthrose du genou, l'effet étant observé plusieurs mois après la fin de la cure [28]. Est-ce dû au stress modéré de la chaleur appliquée (jusqu'à $45^{\circ} \mathrm{C}$ ) ou à d'autres facteurs [29] ?

De nombreuses questions se posent quant à l'utilisation du stress modéré chez l'humain, si son efficacité est avérée. Un stress modéré utilisé à un âge jeune 
représente t-il un stress fort à un âge plus avancé, avec des risques d'effets négatifs? Un stress modéré pour une personne ne risque-t-il pas d'être un stress fort pour une autre? Le problème de l'hétérogénéité des patients se pose donc. Répondre à ces questions implique d'établir un compromis entre les effets positifs attendus ou observés du stress modéré, et les effets négatifs éventuels, comme c'est le cas pour tout traitement. Il est difficile de dire ce que devraient être les paramètres de ce compromis, si ce n'est que bien évidemment le remède ne doit pas être pire que le mal. Quoi qu'il en soit, définir ce compromis est la tâche des médecins mettant en œuvre par exemple un essai clinique randomisé et faisant appel à leur expérience clinique. Le stress modéré peut-il être utilisé seulement en thérapie ou aussi en prévention? Pour reprendre l'exemple de l'arthrose du genou, si l'échauffement local améliore la situation du patient, peut-on imaginer de proposer ce traitement en prévention à partir d'un certain âge ? Si un stress modéré est utilisé en thérapie, utiliser un second stress pour un autre but thérapeutique ne risque-t-il pas de constituer un stress fort? Toutes ces questions soulignent l'ampleur du chemin à parcourir et des études à réaliser. Une étude récente confirme ce concept en montrant que soumettre des souris à une privation de protéines ou d'un acide aminé (le tryptophane) pendant 6 ou 14 jours améliore la survie des animaux après une ischémie-reperfusion rénale [30]. Il est probable que cette privation brutale de protéines place l'organisme dans une situation où il se prépare à résister à des agressions ultérieures, comme attendu face à un stress modéré, et ces résultats ouvrent des perspectives médicales [31].

Le stress modéré est une approche médicale peu invasive qui reste encore peu étudiée. Ainsi, il a été demandé aux auteurs invités à participer à la publication d'un numéro spécial de la revue DoseResponse de donner leur opinion sur l'application possible du stress modéré chez l'humain [32]. Tous ont répondu que cela était possible, même si le débat fut plus ouvert quant à la manière d'utiliser le stress modéré. Peut-être s'agit-il d'une piste à considérer avec attention, au-delà des résultats maintenant connus concernant l'ischémie, afin d'améliorer la vie des personnes âgées? $\diamond$

\section{SUMMARY}

Mild stress as a means to modulate aging:

from fly to human?

Hormesis is the phenomenon by which adaptive responses to low doses of otherwise harmful conditions improve the functional ability of organisms. Some mild stresses have beneficial effects on longevity, aging and resistance to strong stresses (heat or cold shocks, infection) in Drosophila flies. Studies on rodents are indeed scarce but mild stress seems to be effective in humans because, for instance, patients suffering from angina have a higher survival when confronted with a heart attack. A few studies, in less tragic situations however, suggest that mild stress could have positive effects in elderly people. Performing more experiments on the effects of mild stress in humans would help to know whether it could be used in therapy or to improve healthspan of elderly. $\diamond$

\section{CONFLITS D'INTÉRÊTS}

L'auteur déclare n'avoir aucun conflit d'intérêts concernant les données publiées dans cet article.

\section{RÉFÉRENCES}

1. Pérès K. Comment vont nos anciens ? In : Le Bourg $\varepsilon$, ed. Retraites, démographie, santé... vieillir en France aujourd'hui et demain. Paris : Vuibert, $2010: 133-54$.

2. Calabrese $\varepsilon$ ), Baldwin LA. Chemical hormesis: its historical foundation as a biological hypothesis. Hum Exp Toxicol $2000 ; 19: 2-31$.

3. Burton V, Mitchell HK, Young P, Petersen NS. Heat shock protection against cold stress of Drosophila melanogaster. Mol Cell Biol 1988; $8: 3550-2$.

4. Le Bourg $\varepsilon$. Hypergravity in Drosophila melanogaster. In : Le Bourg $\varepsilon$, Rattan SIS, eds. Mild stress and healthy aging. Applying hormesis in aging research and therapy. Berlin : Springer, $2008:$ 43-63.

5. Le Bourg $\varepsilon$. Three mild stresses known to increase longevity in Drosophila melanogaster flies do not increase resistance to oxidative stress. Am J Pharmacol Toxicol 2008 ; 3 : 137-43.

6. Le Bourg E, Massou I, Gobert V. Cold stress increases resistance to fungal infection throughout life in Drosophila melanogaster. Biogerontology 2009 ; $10: 613-25$.

7. Le Bourg $\varepsilon$. Hormesis, aging, and longevity. Biochim Biophys Acta 2009 ; $1790: 1030-9$.

8. Le Bourg $\varepsilon$. Hormetic effects of repeated exposures to cold at young age on longevity, aging and resistance to heat or cold shocks in Drosophila melanogaster. Biogerontology $2007 ; 8$ : 431-44.

9. Le Bourg $\varepsilon$. A cold stress applied at various ages can increase resistance to heat and fungal infection in aged Drosophila melanogaster flies. Biogerontology $2011 ; 12$ : 185-93.

10. Holloszy J0, Smith $\varepsilon K$, Vining M, Adams S. Effect of voluntary exercise on longevity of rats. J Appl Physiol $1985 ; 59: 826-31$.

11. Narath $\varepsilon$, Skalicky M, Viidik A.Voluntary and forced exercise influence the survival and body composition of ageing male rats differently. Exp Geront $2001 ; 36: 1699-711$.

12. Dorner $H$, Stadick J, Platt $D$. Influence of age and treadmill running on motor performance in rats. Arch Geront Geriatr $1997 ; 25: 119-30$.

13. Utsuyama M, Ichikawa M, Konno-Shirakawa A, et al. Retardation of the age-associated decline of immune functions in aging rats under dietary restriction and daily physical exercise. Mech Ageing Dev $1996 ; 91: 219-28$.

14. Loupal G, Url A, Skalicky M, Viidik A. Physical exercise retards the development of chronic nephropathy in the ageing rat as efficiently as food restriction does. Gerontology $2005 ; 51: 83-93$.

15. Ji LL. Physical activity: a strong stimulant for hormesis during aging. In : Le Bourg $\varepsilon$, Rattan SIS, eds. Mild stress and healthy aging. Applying hormesis in aging research and therapy. Berlin : Springer 2008 : 97-114.

16. Holloszy J0, Smith EK. Longevity of cold-exposed rats: a reevaluation of the "rate of living theory". J Appl Physiol 1986; 61 : 1656-60.

17. Paffenbarger RS, Hyde RT, Hsieh CC, Wing AL. Physical activity, other lifestyle patterns, cardiovascular disease and longevity. Acta Med Scand 1986; 711 (suppl) : 85-91.

18. Paffenbarger RS, Kampert JB, Lee IM, et al. Changes in physical activity and other lifeway patterns influencing longevity. Med Sci Sports Exerc $1994 ; 26$ : 857-65.

19. Colcombe S, Kramer AF. Fitness effects on the cognitive function of older adults: a meta-analytic study. Psychol Sci $2003 ; 14$ : 125-30.

20. Abete P, Rengo F. Mild stress in the aging heart: role of ischemic preconditioning. In : Le Bourg $\varepsilon$, Rattan SIS, eds. Mild stress and healthy aging. Applying hormesis in aging research and therapy. Berlin : Springer, 2008 : 139-5.

21. Abete P, Cacciatore F, Testa G, et al. Ischemic preconditioning in the aging heart: from bench to bedside. Ageing Res Rev 2010 ; 9 : 153-62.

22. Prospective Studies Collaboration, Whitlock G, Lewington S, et al. Bodymass index and cause-specific mortality in 900000 adults: collaborative analyses of 57 prospective studies. Lancet 2009 ; 373 : 1083-96.

23. Béjot $Y$, Aboa-Eboulé C, Marie C, Giroud M. Effet neuroprotecteur de l'accident ischémique transitoire. Presse Med 2011 ; 40 : 167-72.

24. Biro S, Masuda A, Kihara T, Tei C. Clinical implications of thermal therapy in lifestyle-related diseases. Exp Biol Med 2003 ; 228 : 1245-9.

25. Dirnagl U, Becker K, Meisel A. Preconditioning and tolerance against cerebral ischemia: from experimental strategies to clinical use. Lancet Neurol $2009 ; 8: 398-412$. 


\section{RÉFÉRENCES}

26. Bordet $\mathrm{R}$, Ouk $\mathrm{T}$, Onténiente $\mathrm{B}$, et al. Ischémie cérébrale. Les pistes thérapeutiques de demain. Med Sci (Paris) $2009 ; 25: 847-54$.

27. Markovic M, Stuhlmeier KM. Short-term hyperthermia prevents activation of proinflammatory genes in fibroblast-like synoviocytes by blocking the activation of the transcription factor NF-kappaB.J Mol Med 2006; $84: 799-801$

28. Forestier $\mathrm{R}$, Desfour $H$, Tessier JM, et al. Spa therapy in the treatment of knee osteoarthritis: a large randomised multicentre trial. Ann Rheum Dis $2010 ; 69: 660-5$

29. Fioravanti A, Cantarini L, Guidelli GM, Galeazzi M. Mechanisms of action of spa therapies in rheumatic diseases: what scientific evidence is there? Rheumatol Int $2011 ; 31: 1-8$.

30. Peng W, Robertson L, Gallinetti J, et al. Surgical stress resistance induced by single amino acid deprivation requires Gcn2 in mice. Sci Transl Med $2012 ; 4$ : 118rall.
31. Mitchell JR, Verweij M, Brand K, et al. Short-term dietary restriction and fasting precondition against ischemia reperfusion injury in mice. Aging Cell $2010 ; 9: 40-53$

32. Le Bourg $\varepsilon$, Rattan SIS. Is hormesis applicable as a pro-healthy aging intervention in mammals and human beings, and how? Introduction to a special issue of Dose-Response. Dose-Response $2010 ; 8$ : 1-3.

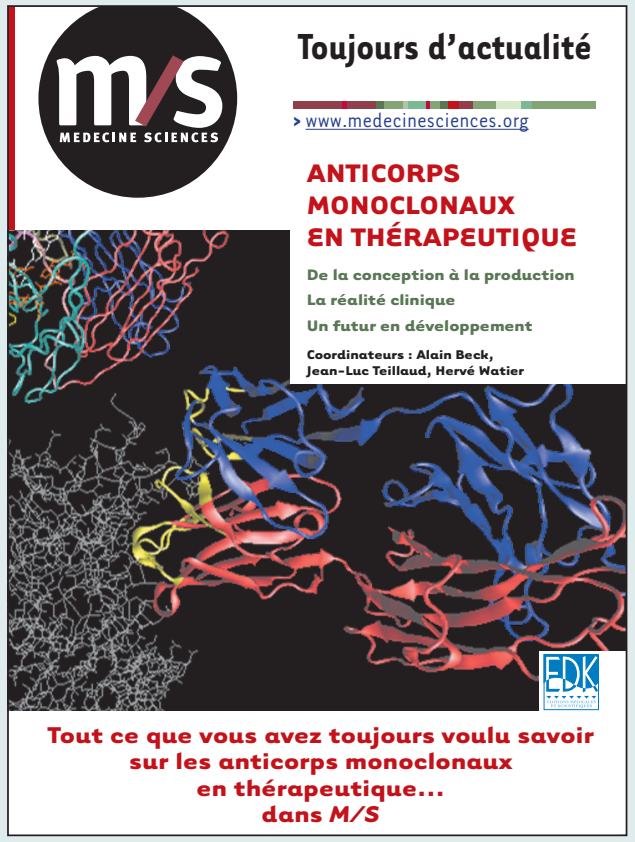

Tout ce que vous avez toujours voulu savoir sur les anticorps monoclonaux en thérapeutique... dans Médecine/Sciences. Pourquoi un numéro spécial de Médecine/Sciences sur les anticorps monoclonaux thérapeutiques? II nous a semblé que le moment était venu de dresser un état des lieux de ces biomédicaments qui prennent désormais une place considérable - et croissante dans les traitements de maladies souvent lourdes et désespérantes. Ce voyage que nous vous proposons à la découverte du monde des anticorps thérapeutiques nous a appris, ou plutôt rappelé, une évidence : les compétences en France sont fortes et nombreuses, qu'elles soient académiques ou industrielles, biotechnologiques ou cliniques. Le paysage français, trop longtemps discret, bruisse désormais de mille initiatives balayant de multiples aspects des anticorps thérapeutiques : études précliniques et cliniques menées avec de nouveaux anticorps dirigés contre des cibles originales, développement de nouveaux formats d'anticorps ou d'anticorps optimisés reposant sur des études structurales et fonctionnelles sophistiquées, recherche active de cibles pertinentes, mise au point de méthodologies de bioproduction, de couplage, etc. L'expansion industrielle rapide de ce champ est un défi que peut et doit relever notre pays, défi tant scientifique qu'économique, avec ses combats pour la propriété intellectuelle et pour l'emploi de nos jeunes scientifiques.

Alain Beck, Jean-Luc Teillaud, Hervé Watier

d) À retourner à EDK, 25, rue Daviel - 75013 Paris, France

Tél. : 0158101905 - Fax : 0143293262 - E-mail : edk@edk.fr

NOM :

Prénom :

Adresse :

Code postal :

Ville :

Pays :

Fonction :

Je souhaite recevoir $\mathbf{M} / \mathbf{S} \mathbf{n}^{\circ} \mathbf{1 2}$ - décembre 2009 (Anticorps monoclonaux en thérapeutique) : $25 €+3 €$ de port $=\mathbf{2 8} € \mathbf{T T C}$ $€$

Par chèque, à l'ordre de $\mathbf{E} \mathbf{D}$ K

Par carte bancaire : $\square$ Visa Eurocard/Mastercard

Carte $n^{\circ}$

Date d'expiration :

$\mathrm{N}^{\circ}$ de contrôle au dos de la carte :

Signature : 